Original Research Paper

\title{
Double Application of Translocator Protein Ligands and RAW Cells Inflammatory Milieu
}

\author{
${ }^{1,2}$ Maria Staykova, ${ }^{3}$ Filomena Mattner, ${ }^{2}$ David Linares and ${ }^{3,4}$ Andrew Katsifis \\ ${ }^{1}$ The John Curtin School of Medical Research, Australian National University, Australia \\ ${ }^{2}$ Neurosciences Research Unit, Canberra Hospital, Australia \\ ${ }^{3}$ Department of PET and Nuclear Medicine, Royal Prince Alfred Hospital, Sydney, Australia \\ ${ }^{4}$ School of Pharmacy, University of Sydney, Australia
}

Article history

Received: 01-08-2016

Revised: $15-08-2016$

Accepted: 16-08-2016

Corresponding Author:

Filomena Mattner

Department of Molecular

Imaging, Royal Prince Alfred

Hospital, Building 63, Level

A7, Missenden Road, NSW

2050, Australia

Email

fmattner@nucmed.rpa.cs.nsw.gov.au

\section{Introduction}

The translocator protein $(18 \mathrm{kDa})$ (TSPO), in conjunction with the voltage dependent anion channel and the adenine nucleotide transporter, forms an integral component of the outer mitochondrial membrane, mediating steroidogenesis, heme biosynthesis, porphyrin and anion transport, apoptosis and cell proliferation (Gavish et al., 1999; Papadopoulos et al., 2006; Rupprecht et al., 2010). This broad spectrum of bioactivities makes it an attractive theurapeutic drug target and a number of TSPO ligands has been synthesised and evaluated for their functional effects on TSPO and development of potential therapeutic agents (Szewczyk and Wojtczak, 2002; Galiegue et al., 2003; Karlstetter et al., 2014; Selvaraj et al., 2015). In addition, the development of radiolabelled TSPO ligands as molecular markers for imaging (Katsifis et al., 2000; Fookes et al., 2008; Pulli and Chen, 2014; Katsifis et al., 2004) helped localizing and monitoring the TSPO upregulation on activated microglia and/or astrocytes which is one of the hallmarks of neuroinflammation and neurodegeneration (Wilms et al., 2003; Girard et al., 2008; Mattner et al., 2011; Daugherty et al., 2013; Mattner et al., 2013).

In this study, the inflammatory milieu of nonactivated and activated macrophage-like cells was tested after a single and double exposure to TSPO ligands. As the ligands affinity and selectivity is dependent on the substitution patterns on the 2-phenyl ring and the acetamide side chains, six novel TSPO ligands were selected to cover a spectrum of chemical structures. The ligands PK11195 and Ro5-4864, originally used in TSPO characterization, were also included.

\section{Materials and Methods}

\section{TSPO Ligands}

The selected TSPO ligands were synthesized as previously described (Katsifis et al., 2000; 2004; Homes et al., 2006; Fookes et al., 2008) and their chemical structures and affinity for TSPO and the central Benzodiazepine Receptor (CBR) are shown in the Fig. 1 The isoquinoline carboxamide PK11195 and the benzodiazepine Ro5-4864 were purchased from Sigma-Aldrich.

\section{RAW 264.7 Cells}

RAW cells $\left(5 \times 10^{5}\right)$ were left to adhere for $5 \mathrm{~h}$ in 8 -well chambers (Nunc Lab-Tek Chamber Slide). Some wells were treated with Lipopolysaccaride (LPS, Calbiochem, La Jolla, CA, USA) and mouse Interferon-gamma (IFN- $\gamma, \mathrm{R}$ and D Systems, Minneapolis, USA) at final concentrations

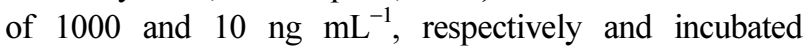
overnight. The culture medium (Staykova et al., 2008) was removed and a fresh medium containing the TSPO ligands at final concentrations of 100,10 or $1 \mathrm{nM}$ was added to non-treated or LPS-IFN- $\gamma$ treated cells. After $12 \mathrm{~h}$ the medium was replaced with a fresh one containing the same ligands and at the same concentrations. Culture supernatants were collected $12 \mathrm{~h}$ later and assayed. 


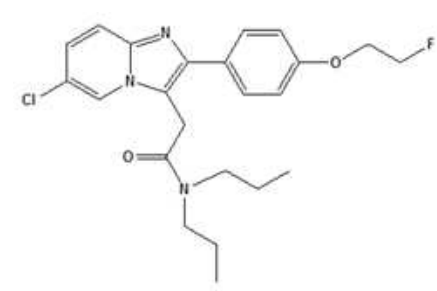

PBR103

$$
\mathrm{K}_{\mathrm{i}}(\mathrm{TSPO})=25.7 \mathrm{nM}
$$

$\mathrm{K}_{\mathrm{i}}(\mathrm{CBR})=1844 \mathrm{nM}$

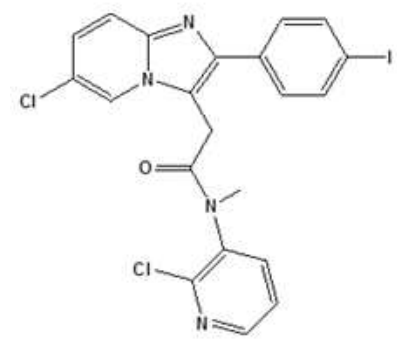

PBR159

$\mathrm{K}_{\mathrm{i}}(\mathrm{TSPO})=3.0 \mathrm{nM}$

$\mathrm{K}_{\mathrm{i}}(\mathrm{CBR})=777 \mathrm{nM}$

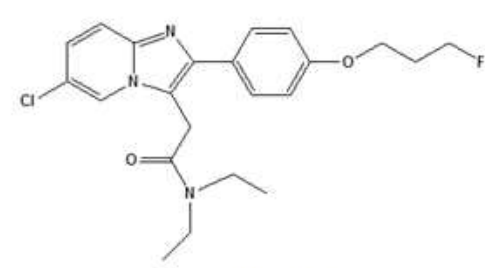

PBR111

$\mathrm{K}_{\mathrm{i}}(\mathrm{TSPO})=3.8 \mathrm{nM}$

$\mathrm{K}_{\mathrm{i}}(\mathrm{CBR})=1588 \mathrm{nM}$

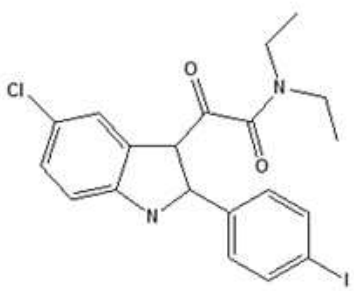

PBR200

$\mathrm{K}_{\mathrm{i}}(\mathrm{TSPO})=4.1 \mathrm{nM}$

$\mathrm{K}_{\mathrm{i}}(\mathrm{CBR})=15652 \mathrm{nM}$

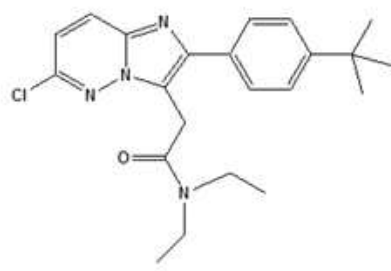

PBR121

$\mathrm{K}_{\mathrm{i}}(\mathrm{TSPO})=15.1 \mathrm{nM}$

$\mathrm{K}_{\mathrm{i}}(\mathrm{CBR})=413 \mathrm{nM}$

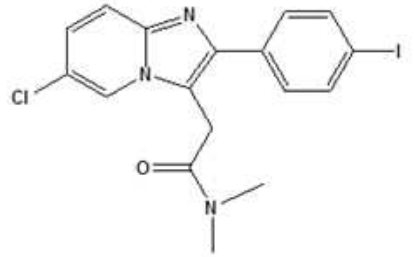

CLINDM

$\mathrm{K}_{\mathrm{i}}(\mathrm{TSPO})=1.2 \mathrm{nM}$

$\mathrm{K}_{\mathrm{i}}(\mathrm{CBR})=232 \mathrm{nM}$

Fig. 1. TSPO ligand chemical structures and binding affinities for TSPO and CBR

\section{Reactive Nitrogen Intermediates (RNI)}

The nitrite was measured colorimetrically after addition of Griess reagent. Nitrate was first converted to nitrite by nitrate reductase in the presence of NADPH (Boehringer Mannheim, Germany) as previously described (Staykova et al., 2008).

\section{Cytokines}

The levels of Tumour Necrosis Factor (TNF), and interleukines (IL: IL-6, IL-10, IL-4) were measured in triplicates in culture supernatants of non-activated and (LPS+IFN- $\gamma$ ) activated RAW cells using ELISA kit (eBioscience, San Diego, Ca, USA) according to the manufacturer's instructions.

\section{Results}

RNI Levels

There was no change in the RNI levels after a single exposure to any ligand at concentrations of 100 and $10 \mathrm{nM}$ as well as after a double treatment of nonactivated RAW cells. However, a double application of all ligands to activated RAW cells resulted in a decrease of the RNI values. At both doses, the strongest effect was observed with PBR103 (70 \pm 2.2 $\mathrm{nM})$, PBR111 (72.6 $\pm 2.8 \mathrm{nM})$ and PBR159 (59.7 \pm 3.2 nM) (Table 1 and Supplementary Table).
Pro-Inflammatory (TNF and IL-6) and AntiInflammatory (IL-4 and IL-10) Cytokines

At the three doses tested a single application of the TSPO ligands had no effect on the levels of these cytokines secreted by non-treated or activated RAW cells.

A double treatment of activated RAW cells with PBR103, PBR111, PBR121, PBR159 PBR200 and CLINDM also resulted in insignificant changes in TNF. In contrast, a double treatment with Ro5-4864 and PK11195 decreased the TNF levels to $2270 \pm 120$ and $2160 \pm 120 \mathrm{pg} \mathrm{mL}^{-1}$, respectively (Table 1 and Supplementary Table). Double exposure of activated RAW cells to all ligands reduced the IL- 6 levels. Most effective was the dose of $1 \mathrm{nM}$ for PBR103, PBR111 and PBR121 (1410 $\left.\pm 100 \mathrm{pg} \mathrm{mL}^{-1}\right)$.

In contrast, double treatment with all ligands resulted in an increase of both anti-inflammatory cytokines. For IL-4 an increase to $0.15 \pm 0.02 \mathrm{pg} \mathrm{mL}^{-1}$ was observed at a dose of $1 \mathrm{nM}$ for ligands PBR103, PBR111, PBR121 and PK11195, while the same dose of $1 \mathrm{nM}$ increased the IL-10 values to $0.19 \pm 0.01 \mathrm{pg} \mathrm{mL}{ }^{-1}$ for PBR103, $0.17 \pm 0.01 \mathrm{pg} \mathrm{mL}^{-1}$ for PBR111, $0.16 \pm 0.02 \mathrm{pg} \mathrm{mL}^{-1}$ for PBR121, $0.17 \pm 0.02 \mathrm{pg} \mathrm{mL}^{-1}$ for PBR159, $0.16 \pm 0.02 \mathrm{pg}$ $\mathrm{mL}^{-1}$ for CLINDM, $0.17 \pm 0.01 \mathrm{pg} \mathrm{mL}^{-1}$ for PK11195 and $0.20 \pm 0.01 \mathrm{pg} \mathrm{mL}^{-1}$ for PBR200. With respect to Ro54864 , most efficient was the dose of $100 \mathrm{nM}(0.17 \pm 0.01$ pg $\mathrm{mL}^{-1}$ ) (Table 1 and Supplementary Table). 
Table 1. Effect of double application of TSPO ligands on RNI and cytokine levels in cultures of activated RAW cells

\begin{tabular}{|c|c|c|c|c|c|c|c|c|c|c|c|c|c|c|}
\hline \multicolumn{3}{|c|}{ RNI } & \multicolumn{3}{|c|}{ TNF } & \multicolumn{3}{|l|}{ IL-6 } & \multicolumn{3}{|l|}{ IL-4 } & \multicolumn{3}{|c|}{ IL-10 } \\
\hline Ligand nM & 100 & 10 & 100 & 10 & 1 & 100 & 10 & 1 & 100 & 10 & 1 & 100 & 10 & 1 \\
\hline PBR103 & $\downarrow 30$ & $\downarrow 30$ & $\uparrow 12$ & 个7 & $\downarrow 20$ & $\downarrow 20$ & $\downarrow 20$ & $\downarrow 36$ & $\uparrow 14$ & $\uparrow 14$ & $\uparrow 27$ & $\uparrow 90$ & $\mathrm{nt}$ & $\uparrow 90$ \\
\hline PBR111 & $\downarrow 27$ & $\downarrow 27$ & 0 & 0 & $\downarrow 13$ & $\downarrow 23$ & $\downarrow 28$ & $\downarrow 36$ & $\uparrow 8$ & $\uparrow 8$ & $\uparrow 27$ & $\uparrow 70$ & $\mathrm{nt}$ & $\uparrow 70$ \\
\hline PBR121 & $\downarrow_{22}$ & $\downarrow 20$ & $\uparrow 9$ & $\uparrow 4$ & $\downarrow 7$ & $\downarrow_{14}$ & $\downarrow 28$ & $\downarrow 35$ & $\uparrow 8$ & $\uparrow 15$ & $\uparrow 27$ & $\uparrow 56$ & $\mathrm{nt}$ & $\uparrow 60$ \\
\hline PBR159 & $\downarrow 40$ & $\downarrow 37$ & $\downarrow 8$ & 0 & $\downarrow 25$ & $\downarrow 20$ & $\downarrow 20$ & $\downarrow_{20}$ & $\uparrow 18$ & $\uparrow 15$ & $\uparrow 15$ & $\uparrow 44$ & $\uparrow 56$ & $\uparrow 67$ \\
\hline PBR200 & $\downarrow 10$ & $\downarrow 10$ & $\downarrow 13$ & $\uparrow 13$ & 0 & 0 & 0 & $\downarrow 14$ & $\uparrow 15$ & $\uparrow 15$ & $\uparrow 15$ & $\uparrow 56$ & $\uparrow 90$ & $\uparrow 100$ \\
\hline CLINDM & $\downarrow 10$ & $\downarrow 15$ & $\downarrow 37$ & $\downarrow 7$ & $\downarrow 20$ & $\downarrow 28$ & $\downarrow 22$ & $\downarrow 28$ & $\uparrow 8$ & $\uparrow 8$ & $\uparrow 15$ & $\uparrow 33$ & $\uparrow 45$ & $\uparrow 56$ \\
\hline Ro5-4864 & $\downarrow 15$ & $\downarrow 15$ & $\downarrow 44$ & $\downarrow 44$ & $\downarrow 40$ & $\downarrow 25$ & $\downarrow 23$ & $\downarrow 23$ & 0 & $\uparrow 8$ & $\uparrow 15$ & $\uparrow 67$ & $\uparrow 56$ & $\uparrow 33$ \\
\hline PK11195 & $\downarrow 10$ & $\downarrow 12$ & $\downarrow 37$ & $\downarrow 35$ & $\downarrow 42$ & $\downarrow 23$ & $\mathrm{nt}$ & $\downarrow 23$ & $\uparrow 8$ & $\uparrow 15$ & $\uparrow 27$ & $\uparrow 44$ & $\uparrow 70$ & $\uparrow 70$ \\
\hline
\end{tabular}

$\downarrow \%$ decrease and $\uparrow \%$ increase in the values in comparison to the ones for activated RAW cells. nt $=$ not tested.

Supplementary Table. Effect of double application of eight TSPO ligands on RNI and cytokine levels in cultures of RAW cells

\begin{tabular}{|c|c|c|c|c|c|c|c|c|c|c|c|c|c|c|}
\hline \multicolumn{3}{|c|}{ RNI nM } & \multicolumn{3}{|c|}{ TNF pg mL $\mathrm{m}^{-1}$} & \multicolumn{3}{|c|}{ IL-6 pg mL ${ }^{-1}$} & \multicolumn{3}{|c|}{ IL-4 pg mL ${ }^{-1}$} & \multicolumn{3}{|c|}{ IL-10 pg/mL $\mathrm{mL}^{-1}$} \\
\hline \multicolumn{3}{|c|}{ Non treated $5.3 \pm 0.5$} & \multicolumn{3}{|c|}{$380 \pm 100$} & \multicolumn{3}{|l|}{$18 \pm 3$} & \multicolumn{3}{|c|}{$0.09 \pm 0.03$} & \multicolumn{3}{|c|}{$0.06 \pm 0.007$} \\
\hline Activated & \multicolumn{2}{|c|}{$99.5 \pm 3.8$} & \multicolumn{3}{|c|}{$3600 \pm 400$} & \multicolumn{3}{|c|}{$2200 \pm 200$} & \multicolumn{3}{|c|}{$0.12 \pm 0.02$} & \multicolumn{3}{|c|}{$0.10 \pm 0.01$} \\
\hline Ligand $\mathrm{nM}$ & 100 & 10 & 100 & 10 & 1 & 100 & 10 & 1 & 100 & 10 & 1 & 100 & 10 & 1 \\
\hline \multirow[t]{2}{*}{ PBR103 } & 70 & 70.1 & 4300 & 3800 & 2900 & 1800 & 1800 & 1410 & 0.14 & 0.14 & 0.15 & 0.19 & $\mathrm{nt}$ & 0.19 \\
\hline & \pm 2 & \pm 2 & \pm 300 & \pm 400 & \pm 500 & \pm 300 & \pm 300 & \pm 200 & \pm .02 & \pm .02 & \pm .02 & \pm .01 & & \pm .01 \\
\hline \multirow[t]{2}{*}{ PBR111 } & 72.6 & 72.6 & 3600 & 3500 & 3000 & 1700 & 1600 & 1410 & 0.13 & 0.13 & 0.15 & 0.17 & $\mathrm{nt}$ & 0.17 \\
\hline & \pm 3 & \pm 3 & \pm 300 & \pm 400 & \pm 500 & \pm 300 & \pm 400 & \pm 200 & \pm .02 & \pm .02 & \pm .01 & \pm .01 & & \pm .01 \\
\hline \multirow[t]{2}{*}{ PBR121 } & 77.6 & 79.6 & 3900 & 3750 & 3350 & 1900 & 1600 & 1410 & 0.13 & 0.14 & 0.15 & 0.16 & $\mathrm{nt}$ & 0.16 \\
\hline & \pm 3 & \pm 3 & \pm 400 & \pm 500 & \pm 500 & \pm 300 & \pm 300 & \pm 100 & \pm .02 & \pm .02 & \pm .02 & \pm .02 & & \pm .02 \\
\hline \multirow[t]{2}{*}{ PBR159 } & 59.7 & 63 & 3300 & 3500 & 2700 & 1800 & 1800 & 1800 & 0.14 & 0.14 & 0.14 & 0.14 & 0.16 & 0.17 \\
\hline & \pm 3 & \pm 3 & \pm 400 & \pm 400 & \pm 500 & \pm 200 & \pm 300 & \pm 300 & \pm . & & \pm .02 & \pm . & \pm .02 & \pm .02 \\
\hline \multirow[t]{2}{*}{ PBR200 } & 89 & 89.2 & 3100 & 4070 & 3600 & 2200 & 2200 & 1900 & 0.14 & 0.14 & 0.14 & 0.16 & 0.19 & 0.2 \\
\hline & \pm 4 & \pm 3 & \pm 300 & \pm 500 & \pm 400 & \pm 300 & \pm 200 & \pm 300 & \pm .02 & \pm .02 & \pm .02 & \pm .01 & \pm .02 & \pm .01 \\
\hline \multirow[t]{2}{*}{ CLINDM } & 90 & 84. & 2260 & 3300 & 2900 & 1500 & 1700 & 1500 & 0.13 & 0.16 & 0.14 & 0.14 & 0.15 & 0.16 \\
\hline & \pm 5 & \pm 3 & \pm 200 & \pm 5 & & \pm 300 & & & \pm . & & & & & \pm .02 \\
\hline \multirow[t]{2}{*}{ Ro5-4864 } & 84.6 & 84.5 & 2270 & 2270 & 2160 & 1600 & 1700 & 17 & 0.1 & 0.14 & 0 . & & 0.16 & 0.13 \\
\hline & \pm 4 & \pm 5 & \pm 120 & \pm 150 & \pm 120 & \pm 300 & \pm 300 & \pm 500 & \pm .02 & \pm .02 & & \pm .01 & & \pm .02 \\
\hline \multirow[t]{2}{*}{ PK11195 } & 90 & 87.1 & 2260 & 2340 & 2090 & 1700 & $\mathrm{nt}$ & 1700 & 0.13 & 0.14 & 0.15 & 0.14 & 0.17 & 0.17 \\
\hline & \pm 5 & \pm 5 & \pm 350 & \pm 300 & \pm 210 & \pm 300 & & \pm 300 & \pm .02 & \pm .02 & \pm .02 & \pm .02 & \pm .02 & \pm .01 \\
\hline
\end{tabular}

\section{Discussion}

A number of reports show an increase in the number of TSPO binding sites in the central and peripheral nervous systems during inflammation as well as neuroprotective effects by TSPO ligands (Ferzaz et al., 2002; Wilms et al., 2003; Mattner et al., 2011; Girard et al., 2012; Daugherty et al., 2013; Mattner et al., 2013; Bae et al., 2014; Morato et al., 2014). Because the principal effector cells in neuroinflammatory and neuro-degenerative disorders are microglia, monocyte-derived macrophages, macrophages in the perivascular space, the choroid plexus and the meninges (Bogie et al., 2014), the use of the RAW cell line (derived from murine macrophages) was an appropriate choice for this study.

In a search of ways to decrease inflammation, and in particular-the neuroinflammation, the effects of two TSPO ligands, Ro5-4864 and PK11195, were extensively studied (Zavala et al., 1990; Ferzaz et al.,
2002; Casellas et al., 2002; Cunningham, 2013; Bae et al., 2014; Morato et al., 2014). However, their antiinflammatory properties were attributed mainly to changes in the steroid synthesis. Recently the TSPO specific ligand vinpocetine was shown to inhibit the production of nitric oxide, IL-1 $\beta$, IL- 6 and TNF- $\alpha$ in microglial cells treated with lipopolysaccharide or exposed to oxygen-glucose deprivation (Zhao et al., 2011). Our in vitro study supports the view that a spectrum of cytokine changes occurs also as a direct result of ligand binding to TSPO. On the other hand, it is difficult to compare our results about no change in RNI and cytokine levels after a single application of Ro5-4864 and PK11195 with the results for rat microglial cells (Choi et al., 2011) and the murine immortalized microglial cell line BV2 (Bae et al., 2014) because the cell activation and the ligands application were the other way round.

While there is a number of pharmacophores with high affinity binding and selectivity to TSPO, the 
structure-affinity relationship models are unable to predict the therapeutic effects. Now we show that double application of nanomolar concentrations of eight TSPO ligands not only has a general inhibitory effect on the pro-inflammatory milleu of activated macrophage-like cells but, more importantly, that the effect do not appear to be related to just the ligand affinity for TSPO or CBR. We believe that these preliminary results on six novel TSPO ligands are encouraging and we intend to increase the number of TSPO ligands tested, as well as the number of applications to non-activated and activated macrophages in order to get a better picture for their potential selective use in regulation of inflammation.

\section{Conclusion}

To our knowledge this is the first report suggesting that multiple applications of different translocator protein ligands may have selective effects on the macrophage inflammatory milieu that do not appear to be related to just the ligand affinity.

\section{Funding Information}

The authors have no support or funding to report.

\section{Author's Contributions}

Maria Staykova: Designed the experiment and performed the RNI tests.

David Linares: Carried out the tests for cytokines.

Filomena Mattner and Andrew Katsifis: Synthesized the six TSPO ligands.

\section{Ethics}

This article is original and contains unpublished material. The corresponding author confirms that all of the other authors have read and approved the manuscript and no ethical issues involved.

\section{References}

Bae, K.R., H.J. Shim, D. Balu, S.R. Kim and S.W. Yu, 2014. Translocator protein $18 \mathrm{kDa}$ negatively regulates inflammation in microglia. J. Neuroimmune. Pharmacol., 9: 424-437. PMID: 24687172

Bogie, J.F., P. Stinissen and J.J. Hendriks, 2014. Macrophage subsets and microglia in multiple sclerosis. Acta Neuropathol., 128: 191-213. DOI: $10.1007 / \mathrm{s} 00401-014-1310-2$

Casellas, P., S. Galiegue and A.S. Basile, 2002. Peripheral benzodiazepine receptors and mitochondrial function. Neurochem. Int., 40: 475-486.

DOI: 10.1016/S0197-0186(01)00118-8
Choi, J., M. Ifuku, M. Noda and T.R. Guilarte, 2011. Translocator protein (18 $\mathrm{kDa}) /$ peripheral benzodiazepine receptor specific ligands induce microglia functions consistent with an activated state. Glia, 59: 219-230. DOI: 10.1002/glia.21091

Cunningham, C., 2013. Microglia and neurodegeneration: The role of systemic inflammation. Glia, 61: 71-90. DOI: $10.1002 /$ glia.22350

Daugherty, D.J., V. Selvaraj, O.V. Chechneva, X.B. Liu and D.E. Pleasure et al., 2013. A TSPO ligand is protective in a mouse model of multiple sclerosis. EMBO Mol. Med., 5: 891-903. DOI: $10.1002 / \mathrm{emmm} .201202124$

Ferzaz, B., E. Brault, G. Bourliaud, J.P. Robert and G. Poughon et al., 2002. SSR180575 (7-ChloroN,N,5-trimethyl-4-oxo-3-phenyl-3,5-dihydro-4Hpyridazino[4,5-b]indole-1-acetamide), a peripheral benzodiazepine receptor ligand, promotes neuronal survival and repair. J. Pharmacol. Exp. Ther., 301: 1067-1078. DOI: 10.1124/jpet.301.3.1067

Fookes, C.J., T.Q. Pham, F. Mattner, I. Greguric and C. Loc'h et al., 2008. Synthesis and biological evaluation of substituted $\left[{ }^{18} \mathrm{~F}\right]$ Imidazo[1,2a]pyridines and $\left[{ }^{18} \mathrm{~F}\right]$ Pyrazolo[1,5-a]pyrimidines for the study of the peripheral benzodiazepine receptor using positron emission tomography. J. Med. Chem., 51: 3700-3712. DOI: 10.1021/jm7014556

Galiegue, S., N. Tinel and P. Casellas, 2003. The peripheral benzodiazepine receptor: A promising therapeutic drug target. Curr. Med. Chem., 10: 1563-1572. PMID: 12871127

Gavish, M., I. Bachman, R. Shoukrun, Y. Katz and L. Veenman et al., 1999. Enigma of the peripheral benzodiazepine receptor. Pharmacol. Rev., 51: 629-650. PMID: 10581326

Girard, C., S. Liu, D. Adams and G. Groyer, 2012. Axonal regeneration and neuroinflammation: Roles for the translocator protein $18 \mathrm{kDa}$. J. Neuroendocrinol., 24: 71-81. DOI: $10.1111 /$ j.1365-2826.2011.02215.x

Girard, C., S. Liu, F. Cadepond, D. Adams and C. Lacroix et al., 2008. Etifoxine improves peripheral nerve regeneration and functional recovery. PNAS, 105: 20505-20510.

DOI: $10.1073 /$ pnas.0811201106

Homes, T.P., F. Mattner, P.A. Keller and A. Katsifis, 2006. Synthesis and in vitro binding of $N, N$-dialkyl2-phenylindol-3-yl-glyoxylamides for the peripheral benzodiazepine binding sites. Bioorg. Med. Chem., 14: 3938-3946. DOI: 10.1016/j.bmc.2006.01.039

Karlstetter, M., C. Nothdurfter, A. Aslanidis, K. Moeller and F. Horn et al., 2014. Translocator protein (18 $\mathrm{kDa}$ ) (TSPO) is expressed in reactive retinal microglia and modulates microglial inflammation and phagocytosis. J. Neuroinflammat., 11: 3-3.

DOI: $10.1186 / 1742-2094-11-3$ 
Katsifis, A., F. Mattner, B. Dikic and V. Papazian, 2000. Synthesis of substituted $\left[{ }^{123} \mathrm{I}\right]$ imidazo[1,2$\alpha]$ pyridines as potential probes for the study of the peripheral benzodiazepine receptors using SPECT. Radiochim Acta, 88: 229-232. DOI: $10.1524 /$ ract.2000.88.3-4.229

Katsifis, A., G. Barlin, F. Mattner and B. Dikic, 2004. Synthesis of $\left[{ }^{123} \mathrm{I}\right]$ iodine labelled imidazo[1,2-b] pyridazines as potential probes for the study of peripheral benzodiazepine receptors using SPECT. Radiochimica Acta, 92: 305-309. DOI: $10.1524 /$ ract.92.4.305.35581

Mattner, F., D. Bandin, M. Staykova, P. Berghofer and M.C. Gregoire et al., 2011. Evaluation of $\left[{ }^{123} \mathrm{I}\right]-$ CLINDE as a potent SPECT radiotracer to assess the degree of astroglia activation in cuprizoneinduced neuroinflammation. Eur. J. Nucl. Med. Mol. Imag., 38: 1516-1528.

DOI: $10.1007 / \mathrm{s} 00259-011-1784-2$

Mattner, F., M. Staykova, P. Berghofer, H.J. Wong and S. Fordham et al., 2013. Central nervous system expression and pet imaging of the translocator protein in relapsing-remitting experimental autoimmune encephalomyelitis. J. Nucl. Med., 54: 291-298. DOI: 10.2967/jnumed.112.108894.

Morato, L., E. Bertini, D. Verrigni, M. Ruiz and I. Ferrer et al., 2014. Mitochondrial dysfunction in central nervous system white matter disorders. Glia, 62: 1878-1894. PMID: 24865954

Papadopoulos, V., M. Baraldi, T.R. Guilarte, T.B. Knudsen and J.J. Lacapère et al., 2006. Translocator protein $(18 \mathrm{kDa})$ : New nomenclature for the peripheral-type benzodiazepine receptor based on its structure and molecular function. Trends Pharmacol. Sci., 27: 402-409. DOI: 10.1016/j.tips.2006.06.005

Pulli, B. and J.W. Chen, 2014. Imaging neuroinflammationfrom bench to bedside. J. Clin. Cell Immunol., 5: 266-266. DOI: 10.4172/2155-9899.1000226
Rupprecht, R., V. Papadopoulos, G. Rammes, T.C. Baghai and J. Fan et al., 2010. Translocator protein (18 $\mathrm{kDa}$ (TSPO) as a therapeutic target for neurological and psychiatric disorders. Nat. Rev. Drug. Discov., 9: 971-988. DOI: $10.1038 / \mathrm{nrd} 3295$

Selvaraj, V., D.M. Stocco and L.N. Tu, 2015. Minireview: Translocator Protein (TSPO) and steroidogenesis: A reappraisal. Mol. Endocrinol., 29: 490-501. PMID: 25730708

Staykova, M., D. Linares, S. Fordham, J. Paridaen and D.O. Willenborg, 2008. The innate immune response to adjuvants dictates the adaptive immune response to autoantigens. J. Neuropathol. Exp. Neurol., 67: 543-554.

DOI: $10.1097 /$ NEN.0b013e31817713cc

Szewczyk, A. and L. Wojtczak, 2002. Mitochondria as a pharmacological target. Pharmacol. Rev., 54: 101-127. PMID: 11870261

Wilms, H., J. Claasen, C. Rohl, J. Sievers and G. Deuschl et al., 2003. Involvement of benzodiazepine receptors in neuroinflammatory and neurodegenerative diseases: Evidence from activated microglial cells in vitro. Neurobiol. Dis., 14: 417-424. DOI: 10.1016/j.nbd.2003.07.002

Zavala, F., V. Taupin and B. Descamps-Latscha, 1990. In vivo treatment with benzodiazepines inhibits murine phagocyte oxidative metabolism and production of interleukin 1, tumor necrosis factor and interleukin-6. J. Pharmacol. Exp. Ther., 255: 442-450. J. Pharmacol. Exp. Ther., 255: 442-450. PMID: 1978727

Zhao, Y., J. Yu, Q. Li, C. Ma and C. Lu et al., 2011. TSPO-specific ligand vinpocetine exerts a neuroprotective effect by suppressing microglial inflammation. Neuron Glia Biol., 7: 187-197. DOI: $10.1017 / \mathrm{S} 1740925 \mathrm{X} 12000129$ 